\title{
Dynamic observation of 5-fluorouracil-induced myocardial injury and mitochondrial autophagy in aging rats
}

\author{
YUANYANG LI ${ }^{1,2^{*}}$, YUFAN ZHANG ${ }^{2,3^{*}}$, XIANGZHONG ZHOU ${ }^{4}$, \\ XIANGHONG LEI ${ }^{5}$, XINHANG LI ${ }^{1,2}$ and LIPING WEI ${ }^{2}$ \\ ${ }^{1}$ School of Graduate Studies, Tianjin University of Traditional Chinese Medicine, Tianjin 301677; \\ ${ }^{2}$ Department of Cardiology, Tianjin Union Medical Center, Nankai University Affiliated Hospital, \\ Tianjin 300121; ${ }^{3}$ School of Graduate Studies, Tianjin Medical University, Tianjin 300070; \\ ${ }^{4}$ Department of Cardiology, Tianjin Da Gang Hospital, Tianjin 300270; ${ }^{5}$ Department of Ultrasound, \\ Tianjin Union Medical Center, Nankai University Affiliated Hospital, Tianjin 300121, P.R. China
}

Received August 1, 2020; Accepted July 6, 2021

DOI: $10.3892 /$ etm.2021.10886

\begin{abstract}
Patients treated with 5-fluorouracil (5-FU) can develop rare but potentially severe cardiac effects, including cardiomyopathy, angina pectoris, heart failure and cardiogenic shock. The specific pathologies and underlying mechanisms are yet to be fully understood. The results of previous studies have indicated that mitochondrial autophagy is widely detected in many angiocardiopathies. In the present study, the dynamic changes in the homeostasis of mitochondrial injury and autophagy were observed in rats treated with 5-FU for different durations. A corresponding control group and a 5-FU model group were established in groups of Sprague-Dawley rats aged 2 and 18 months, and the myocardial enzyme levels were determined at different time points. At 2 weeks post-model establishment, cardiac ultrasound and myocardial histological staining were performed, cardiomyocyte apoptosis and myocardial mitochondrial function were assessed, and mitochondrial ultrastructure was examined. In addition, the expression levels of autophagy-related proteins were evaluated in the 18-month-old rats on days 7 and 14 of 5-FU administration. The experimental results demonstrated that 5-FU induced an elevation in the levels of myocardial enzymes, as well as changes in the cardiac structure and function, and that these changes were more prominent over longer drug durations. In addition, 5-FU decreased the levels of
\end{abstract}

Correspondence to: Professor Liping Wei, Department of Cardiology, Tianjin Union Medical Center, Nankai University Affiliated Hospital, 190 Jieyuan Road, Hongqiao, Tianjin 300121, P.R. China

E-mail: weilipingme@163.com

*Contributed equally

Key words: 5-fluorouocil, cardiotoxicity, aging, mitochondrial autophagy myocardial mitochondrial ATP and mitochondrial membrane potential, and aggravated myocardial fibrosis and cardiomyocyte apoptosis compared with those observed in the untreated control group, treated with the same volume of saline as 5-FU in the 5-FU group. These injuries were particularly evident in aging rats. Notably, 5-FU increased the expression levels of myocardial mitochondrial autophagy-related proteins, and electron microscopy revealed a more severe autophagic state in the model groups compared with that in the control groups. In conclusion, 5-FU induced myocardial mitochondrial damage, the degree of which was more severe in aging rats compared with that in young rats. The mitochondrial autophagy induced by 5 -FU was excessive, and the degree of autophagy was aggravated with increased 5-FU administration time.

\section{Introduction}

5-Fluorouracil (5-FU) is an antimetabolic chemotherapeutic drug that is widely used in the clinic (1). 5-FU is widely used in the treatment of tumors in various parts of the body, such as breast, liver and ovarian cancer, and has also been used for the treatment of gastrointestinal tumors such as gastric cancer (2). 5-FU, as a commonly used chemotherapy drug for digestive tract tumors, is very important for the treatment of both elderly patients who cannot tolerate surgery and need chemotherapy, and patients who have undergone surgery $(3,4)$. However, in addition to its antitumor effects, patients may experience adverse reactions in various organs, including tumor-associated cardiovascular disease $(5,6)$. Clinical data on the cardiotoxicity of antimetabolic chemotherapeutic drugs has revealed that elderly patients, particularly those with cardiovascular disease, exhibit a higher incidence of cardiotoxicity and experience more adverse reactions than younger patients without cardiovascular disease $(7,8)$. Therefore, the role of cardiotoxicity following the use of chemotherapeutic drugs requires further investigation, particularly in elderly patients with cancer. Current investigations aim to minimize the heart damage experienced due to chemotherapy in patients who undergo tumor-suppressing treatments $(9,10)$. 
The results of previous studies have demonstrated the pathological mechanisms underlying 5-FU-induced cardiotoxicity, including oxidative stress, cytotoxic effects, arterial vasospasm outside the cardiac vasculature, apoptosis, autoimmune reactions and changes in vascular endothelial function (11-13). Lemaire et al (14) revealed that 5-FU is converted to a number of metabolites, which leads to mitochondrial swelling and a decrease in mitochondrial membrane permeability (14). Eskandari et al (12) demonstrated that 5-FU induced oxidative stress and decreased mitochondrial function in the myocardium, leading to apoptosis of cardiomyocytes and cardiotoxicity in rabbits (12).

The results of a previous study indicated that mitochondrial damage in cardiomyocytes is the basis of 5-FU cardiotoxicity (15). Damaged mitochondria are degraded or eliminated by autophagy to meet the metabolic needs of the cells, for organelle regeneration and to maintain cell homeostasis (16). Insufficient or excessive mitochondrial autophagy causes myocardial damage $(17,18)$. However, the mechanisms underlying 5-FU-induced myocardial mitochondrial damage and mitochondrial autophagy in aging organisms remain to be elucidated. Thus, the dynamic changes induced by $5-\mathrm{FU}$ on myocardial mitochondrial damage were investigated in the aging rat model in the present study, and the molecular mechanisms underlying cardiotoxicity were assessed from the perspective of mitochondrial autophagy imbalance.

\section{Materials and methods}

Animal model and treatment protocols. A total of 60 male

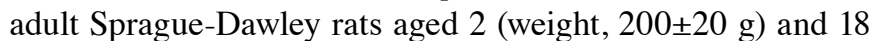
(weight, 600 $\pm 50 \mathrm{~g}$ ) months were purchased from Beijing Vital River Laboratory Animal Technology Co., Ltd.; Charles River Laboratories, Inc. and used in the present study. The animals were kept under a 12-h light/dark cycle in an environment with a temperature of $20^{\circ} \mathrm{C}$ and a humidity of $50 \%$, with free access to food and water, and experiments were performed after 1 week of acclimatization. All animals were used in accordance with ethics guidelines, respecting animal welfare and minimizing discomfort. The experimental protocol was approved by the Animal Care and Use Committee of Tianjin Union Medical Center (approval no. 2020-B03; Tianjin, China).

The 2-month-old rats were randomly divided into a young age control ( $\mathrm{YC} ; \mathrm{n}=10$ ) and young age model ( $\mathrm{YM} ; \mathrm{n}=10)$ groups. The 18-month-old rats were randomly divided into an aging control (AC; $n=20)$ and aging model (AM; $=20)$ groups. The 5-FU-induced cardiotoxicity model groups were administered an intraperitoneal (i.p.) injection of $25 \mathrm{mg} / \mathrm{kg} 5-\mathrm{FU}$ (Shanghai Xudong Haipu Pharmaceutical Co., Ltd.) every other day for 1 or 2 consecutive weeks. The control groups received the same volume of saline at the same time points as the 5-FU group.

A total of 6 rats were randomly selected from each group and 600-800 $\mu \mathrm{l}$ blood was collected from the tail vein of these rats on days 0,7 and 14 . The blood was used for the detection of creatine kinase isoenzyme (CK-MB) and cardiac troponin I (cTnI). On day 14 , all remaining rats underwent small animal cardiac ultrasound evaluation. On day 15 , the rats were anesthetized following an i.p. injection with $3 \%$ pentobarbital sodium solution at an anesthetic dose of $40 \mathrm{mg} / \mathrm{kg}(19,20)$, blood was collected from the abdominal aorta, and rats were sacrificed by cervical dislocation. Fresh myocardial tissues were collected and washed with cold saline. A portion of each tissue was used for mitochondrial extraction to determine the levels of ATP, mitochondrial membrane potential (MMP) and specific mitochondrial proteins. The remaining tissues were stored at $-80^{\circ} \mathrm{C}$ or fixed with $4 \%$ paraformaldehyde for $48 \mathrm{~h}$ and embedded in paraffin.

In order to further evaluate the degree of myocardial mitochondrial autophagy following different 5-FU treatment durations, the aged rats were divided into two subgroups. Following the first week of 5-FU administration, 8 rats were randomly selected from the aged group and euthanized, and the remaining rats were euthanized after the second week of 5-FU administration. Heart tissues were collected, and mitochondrial proteins were extracted for the subsequent analysis of autophagy-related protein expression. The overall experimental process is displayed in Fig. 1.

Measurement of serum CK-MB and cTnI levels. A total of 6 rats were randomly selected from each group and blood was collected from the tail vein on days 0,7 and 14 . Following incubation at room temperature for $30 \mathrm{~min}$, all blood samples were centrifuged at $4^{\circ} \mathrm{C}$ and $3,000 \mathrm{x}$ g for $15 \mathrm{~min}$ to separate the serum. The serum levels of CK-MB (cat. no. E-EL-R1327c) and cTnI (cat. no. E-EL-R1253c) were subsequently detected by ELISA (Elabscience Biotechnology, Inc.). The optical density was determined using a microplate reader at $450 \mathrm{~nm}$, a standard curve was plotted and the sample index concentration was calculated (CK-MB, $\mathrm{y}=135.64 \mathrm{x}^{2}+700.01 \mathrm{x}-40.521$, $\mathrm{R}^{2}=0.999$; cTnI, $\left.\mathrm{y}=3984.1 \mathrm{x}^{2}-330.74 \mathrm{x}+27.441, \mathrm{R}^{2}=0.996\right)$.

Echocardiography. On day 14 of 5-FU administration, rats were anesthetized by an i.p. injection with $3 \%$ pentobarbital sodium solution at a dose of $40 \mathrm{mg} / \mathrm{kg}$. The fur was removed from the chest and the rats were fixed on a platform with a horizontal tilt of $15^{\circ}$. Short-axis m-type echocardiography was used to determine left ventricular internal dimension in diastole (LVID-d), left ventricular internal dimension in systole (LVID-s), left ventricular ejection fraction (LVEF; \%) and left ventricular shortening fraction (LVFS; \%) using a Vevo2100 high-resolution imaging system (VisualSonics, Inc.).

Heart weight index (HWI) assessment. Total heart tissue was obtained and washed with cold PBS, and excess water was removed using an absorbent paper towel. The adipose tissue was removed, the aorta was separated from the atrium and the weight of each heart was determined in $\mathrm{mg}$. HWI was defined as the ratio of heart weight to body weight $(\mathrm{mg} / \mathrm{g})$.

Measurement of ATP content. Fresh myocardial tissue (20 mg) was obtained, added to pyrolysis liquid (cat. no. S0027-4; Beyotime Institute of Biotechnology), homogenized on ice and centrifuged at $4^{\circ} \mathrm{C}$ for $5 \mathrm{~min}$ at $12,000 \times \mathrm{g}$ to obtain the supernatant. Standard curves were prepared by diluting the standard ATP solution to different concentrations $(0.01,0.03$, $0.1,0.3,1,3$ and $10 \mu \mathrm{M}$ ) using an enhanced ATP assay kit (cat. no. S0027; Beyotime Institute of Biotechnology) according to the manufacturer's instructions. A working concentration of ATP detection solution (1X) was produced, and $100 \mu \mathrm{l}$ was added to an opaque 96-well plate. In order to consume the ATP in the detection solution, the 96-well plate was placed 


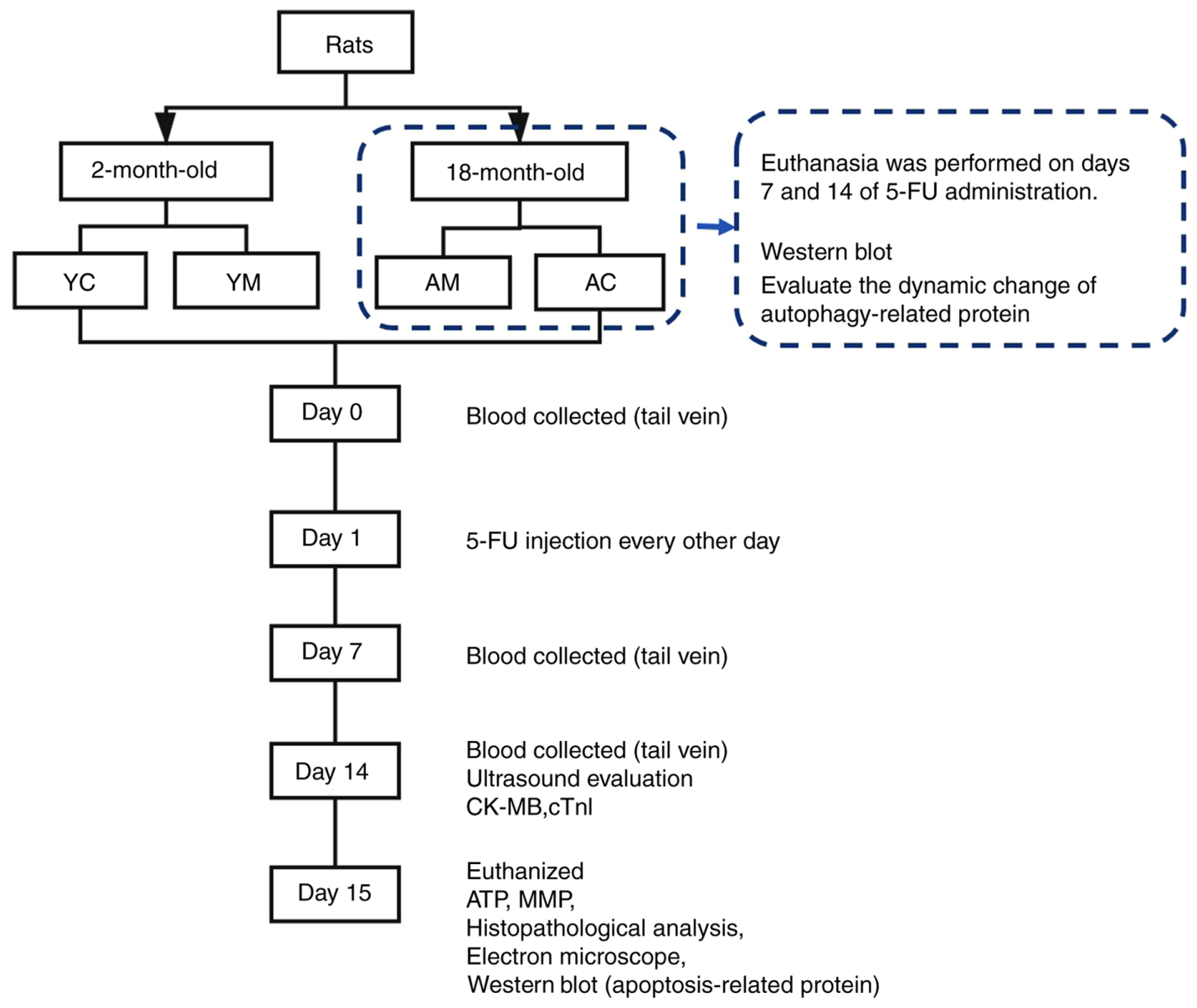

Figure 1. Flow diagram of the experimental process. YC, young age control group; YM, young age model group; AC, aging control group; AM, aging model group; 5-FU, 5-fluorouracil; CK-MB, creatine kinase isoenzyme; cTnI, cardiac troponin I; ATP, adenosine 5'-triphosphate; MMP, mitochondrial membrane potential.

at room temperature for $5 \mathrm{~min}$, thus reducing the background fluorescence. After the ATP background was eliminated, the samples were added to the 96 -well plate. The relative light unit value was determined using a luminometer (Multifunctional Microplate Absorbance Reader; Bio-Rad Laboratories, Inc.). The protein concentration of the homogenates was determined using a BCA protein kit and the final ATP concentration was converted to $\mathrm{nmol} / \mathrm{mg}$ protein for subsequent quantification.

MMP assay. Fresh myocardial tissue $(20 \mathrm{mg})$ was obtained and minced with scissors, prechilled for 3 min using cold PBS, and centrifuged at $4^{\circ} \mathrm{C}$ for $20 \mathrm{sec}$ at $600 \mathrm{x}$ g to obtain the precipitate. Tissues were subsequently prechilled for $20 \mathrm{~min}$ using Trypsin-EDTA Solution (cat. no. C3606-3; Beyotime Institute of Biotechnology) and centrifuged at $4^{\circ} \mathrm{C}$ for $20 \mathrm{sec}$ at $600 \mathrm{x} \mathrm{g}$ to obtain the precipitate. The mitochondria were extracted using a mitochondrial separation reagent (cat. no. C3606-2; Beyotime Institute of Biotechnology), homogenized on ice and centrifuged at $4^{\circ} \mathrm{C}$ at $12,000 \mathrm{x}$ g. This treatment was repeated twice. JC-1 buffer (1X) was prepared using an MMP assay kit with JC-1 (cat. no. C2006; Beyotime Institute of Biotechnology), and $10 \mathrm{mM}$ carbonyl cyanide m-chlorophenylhydrazone was diluted to $10 \mu \mathrm{M}$ for use as a positive control. JC-1 detection fluid (1X) and the samples were added to a 96-well light-proof plate at a ratio of 9:1 to load the purified mitochondria with JC-1. A fluorescence microplate reader was preheated, and the excitation and emission values were set to 490 and $530 \mathrm{~nm}$, respectively, to detect the JC-1 monomer and 525 and $590 \mathrm{~nm}$, respectively, for the polymer. The relative ratio of red and green fluorescence was used to determine the proportion of mitochondrial depolarization.

Histopathological analysis. Left ventricular tissues were fixed with $4 \%$ paraformaldehyde at room temperature for $48 \mathrm{~h}$. Following dehydration with 95 and $100 \%$ ethanol, tissues were incubated in xylene, embedded in paraffin and cut into $4-\mu \mathrm{m}$ sections. Sections were stained with hematoxylin for $5 \mathrm{~min}$, followed by eosin for $5 \mathrm{~min}$ at room temperature. H\&E trichrome staining was subsequently performed. Sections were stained with Weigert iron hematoxylin solution (cat. no. G1340; Beijing Solarbio Science and Technology Co., Ltd.) for $5 \mathrm{~min}$, then differentiated with $1 \%$ hydrochloric acid for $1 \mathrm{~min}$ and rinsed for $1.5 \mathrm{~h}$. Ponceau stain was added for $7 \mathrm{~min}$, and distilled water was used for washing. The sections were treated with a $1 \%$ aqueous solution of phosphomolybdic acid for $\sim 5 \mathrm{~min}$ and counterstained with an aniline blue solution for $5 \mathrm{~min}$. Finally, the sections were treated with $1 \%$ glacial acetic acid for $1 \mathrm{~min}$. After staining, the slices were dehydrated with different concentrations of ethanol and cleared with $100 \%$ xylene. Masson's trichrome staining were performed. All sections were imaged using a light microscope (magnification, x200 and x400). Histopathological analysis was performed 
in six randomly selected regions from each section. ImageJ software (version 1.8.0; National Institutes of Health) was used for image analysis, and collagen volume fraction (CVF) was calculated as the left ventricular collagen area/field area.

TUNEL apoptosis analysis. Apoptosis analysis was conducted using the TUNEL apoptosis detection kit (cat. no. C1091; Beyotime Institute of Biotechnology). Sections were dewaxed for $40 \mathrm{~min}$ at $70^{\circ} \mathrm{C}$, and incubated in xylene for $10 \mathrm{~min}, 100 \%$ ethanol for $5 \mathrm{~min}, 100 \%$ ethanol for $2 \mathrm{~min}, 90 \%$ ethanol for $2 \mathrm{~min}, 80 \%$ ethanol for $2 \mathrm{~min}, 70 \%$ ethanol for $2 \mathrm{~min}$ and washed in distilled water. Following dewaxing and rehydration, the paraffin sections were treated with proteinase K (cat. no. ST352; Beyotime Institute of Biotechnology) and incubated at room temperature for 15 min. Sections were subsequently incubated in Enhanced Endogenous Peroxidase Blocking Buffer (cat. no. P0100B; Beyotime Institute of Biotechnology) at room temperature for $15 \mathrm{~min}$, followed by washing three times for $10 \mathrm{~min}$ with PBS. The negative control samples were treated with 1X Reaction Buffer (cat. no. C-1082-2; Beyotime Institute of Biotechnology) instead of TUNEL Reaction mixture, and the positive control sections were treated with DNase I (cat. no. C-1082-1; Beyotime Institute of Biotechnology), and incubated at $25^{\circ} \mathrm{C}$ for $15 \mathrm{~min}$ to generate double-stranded DNA breaks. Following washing with PBS three times at room temperature for $5 \mathrm{~min}$ each, 3,3'-diaminobenzidine substrate solution was added, and the sections were incubated at $37^{\circ} \mathrm{C}$ for $5 \mathrm{~min}$ prior to signal detection. The samples were counterstained with hematoxylin at room temperature for $5 \mathrm{~min}$. The plates were sealed using gelatin and glycerinum, and imaged under a light microscope (magnification, x400), followed by analysis using Image J software (version, 1.8.0; National Institutes of Health). A total of six random regions were selected from each section for counting and immunohistochemical scoring, according to the following equation: IHS $=\mathrm{A} \times \mathrm{B}$, where $\mathrm{A}$ is the score of positive cells $(0,0-1 ; 1$, $1-10 ; 2,10-50 ; 3,50-80$; and 4, 80-100\%) and $\mathrm{B}$ is the color intensity score ( 0 , negative; 1 , weakly positive; 2 , positive; and 3 , strongly positive) (21).

Electron microscopic observation of the mitochondrial ultrastructure. Fresh myocardial tissues were collected (volume, $<1 \mathrm{~mm}^{3}$ ) and fixed in $2.5 \%$ glutaraldehyde fixation fluid (in $0.1 \mathrm{~mol} / 1$ phosphate buffer) at $4^{\circ} \mathrm{C}$ for $24 \mathrm{~h}$. Following washing three times for $15 \mathrm{~min}$ with $\mathrm{PBS}$, the samples were fixed in $1 \%$ osmium fixative solution at $4^{\circ} \mathrm{C}$ for $2 \mathrm{~h}$, dehydrated two times for $15 \mathrm{~min}$ in a graded ethanol series $(50,70,80,90$ and $100 \%)$, embedded in epoxy resin at $37^{\circ} \mathrm{C}$ overnight under a vented hood and subsequently cut into 70 -nm sections. The ultrathin sections were stained with lead citrate and uranium acetate at room temperature for $15 \mathrm{~min}$, and observed and photographed under a JEM-1200 transmission electron microscope (JEOL Ltd.; magnification, x10,000 and x20,000). Subsequently, a total of three samples were collected from each group, six regions were selected from each section for histopathological analysis, and semi-quantitative analysis was conducted according to the Flameng classification system using ImageJ (version, 1.8.0; National Institutes of Health) (22).
Protein extraction, quantification and western blotting. Samples of fresh myocardial tissue (30 mg/sample) were collected, and the mitochondrial protein was extracted using the Tissue Mitochondrial Isolation kit (cat. no. C3606; Beyotime Institute of Biotechnology) according to the manufacturer's instructions. The protein concentrations were determined using a BCA protein assay kit (cat. no. P0010S; Beyotime Institute of Biotechnology), and the samples were mixed with $5 \mathrm{X}$ loading buffer for denaturation at $95^{\circ} \mathrm{C}$ for $10 \mathrm{~min}$. A total of $30 \mu \mathrm{g}$ protein per sample was isolated by SDS-PAGE on 10 or $15 \%$ gels and transferred to a PVDF membrane. The membrane was subsequently blocked with blocking solution (PBST; 1X; cat. no. P0226; Beyotime Institute of Biotechnology) for $15 \mathrm{~min}$ at room temperature and incubated with the appropriate primary antibody diluted in TBS-Tween-20 (1\% Tween-20) at $4^{\circ} \mathrm{C}$ overnight. Following washing three times for $10 \mathrm{~min}$ with TBS-T, the membrane was incubated with a horseradish peroxidase (HRP)-conjugated secondary antibody, (1:10,000; cat. no. 2722564; ProteinTech Group, Inc.) at room temperature for $90 \mathrm{~min}$, and visualization was performed using luminol reagent (cat. no. P0018AM; Beyotime Institute of Biotechnology). $\beta$-actin was used as a loading control. The primary antibodies used in the present study were as follows: Anti- $\beta$-actin (1:1,000; 4970s; Cell Signaling Technology, Inc.), Anti-Bax (1:10,000; cat. no. 50599-2-lg; ProteinTech Group, Inc.), anti-Bcl-2 (1:3,000; cat. no. 26593-1-AP; ProteinTech Group, Inc.), anti-beclin-1 (1:1,000; \#3738 Cell Signaling Technology, Inc.), anti-Parkin (1:1,000; \#2132 Cell Signaling Technology, Inc.), anti-microtubule-associated protein 1A/1B-light chain 3 (LC3) A/B (1:1,000; \#12741 Cell Signaling Technology, Inc.), anti-PTEN-induced putative kinase protein 1 (PINK-1; 1:500; cat. no. 23274-1-AP; ProteinTech Group, Inc.) and anti-cytochrome c oxidase subunit 4 (COX-IV; 1:1,000; \#4844 Cell Signaling Technology, Inc.).

Statistical analysis. The data were statistically analyzed using SPSS 23.0 (IBM Corp.), and the figures were generated using GraphPad 5.0 (GraphPad Software, Inc.). Normally distributed data are presented as the mean \pm standard deviation. Comparisons among groups were analyzed by one-way ANOVA, and Tukey's post hoc test was used for multiple comparisons. In cases of non-normality, the non-parametric Kruskal-Wallis test followed by Bonferroni post hoc analysis was used for multiple testing. Repeated measurement data were analyzed by repeated measures multi-factor ANOVA and means by LSD test. $\mathrm{P}<0.05$ was considered to indicate a statistically significant difference.

\section{Results}

5-FU induces weight loss and myocardial injury in rats. To evaluate the extent of myocardial damage induced by 5 -FU, a myocardial toxicity model was established in rats aged 2 and 18 months. Notably, the baseline body weight was inconsistent between rats of different ages; thus, the difference between weight gain and loss was used as the evaluation index. The results of the present study demonstrated that 5-FU stimulation significantly reduced the rat body weight compared with that in the model groups. The AM group experienced weight loss from day 3 of 5-FU administration, whereas the YM 

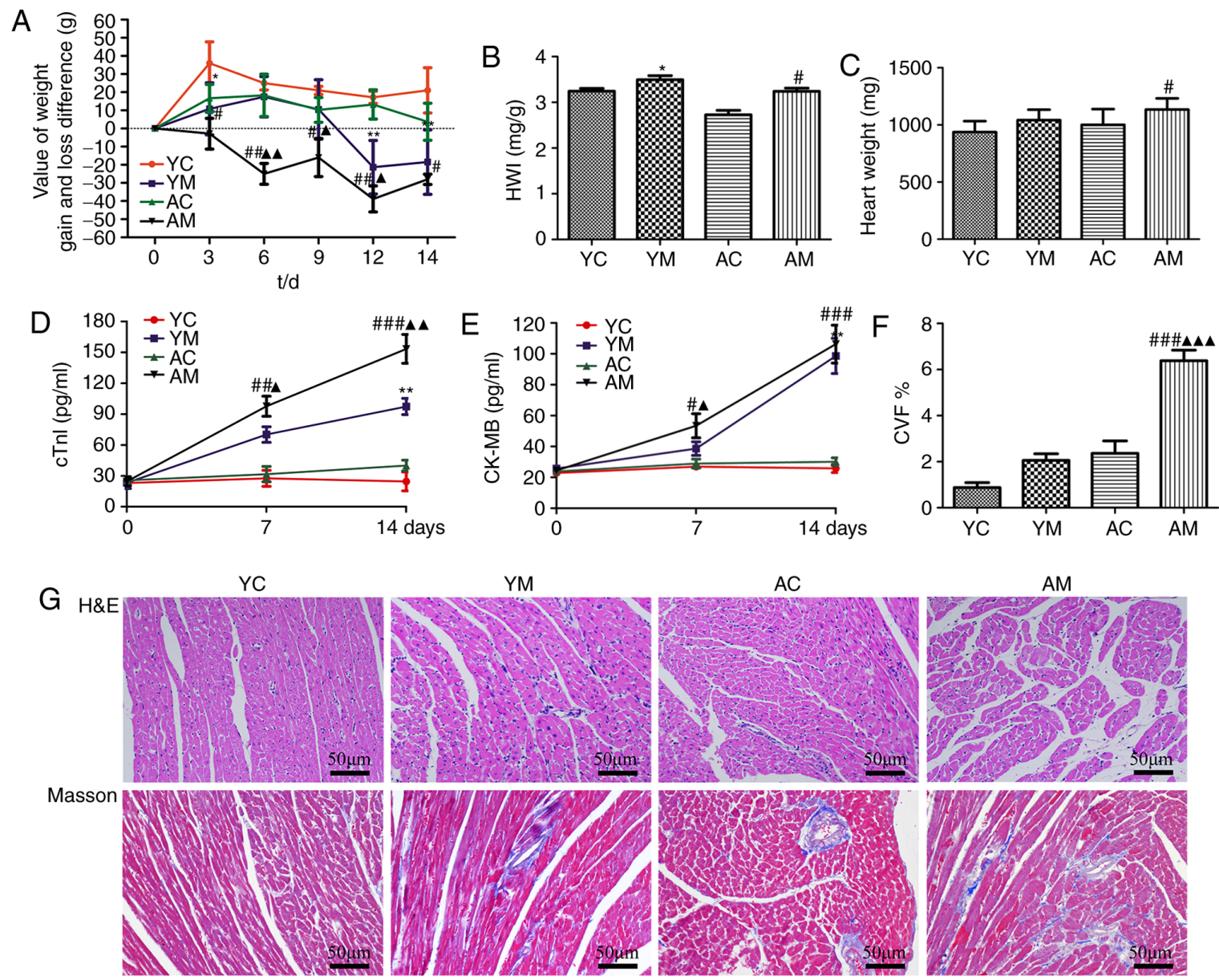

Figure 2. 5-FU induces weight loss and myocardial injury in rats. (A) Variation in weight gain and loss in each group at different time points during 5-FU administration $(\mathrm{n}=8)$. Comparison of $(\mathrm{B}) \mathrm{HWI}$ and $(\mathrm{C})$ heart weight in each group $(\mathrm{n}=6)$. ELISA was used to examine the levels of myocardial enzymes in rat myocardial tissues, (D) cTnI and (E) CK-MB (n=6). (F) CVF of myocardial tissues was determined by quantification of the left ventricular collagen area/field area $(n=6)$. $(\mathrm{G}) \mathrm{H} \& \mathrm{E}$ and Masson's trichrome staining of cardiac tissues (magnification, $\mathrm{x} 200$ ). Blue staining represents fibrosis. ${ }^{*} \mathrm{P}<0.05$ and ${ }^{* *} \mathrm{P}<0.01 \mathrm{vs}$. $\mathrm{YC}$; ${ }^{\#} \mathrm{P}<0.05,{ }^{\# \#} \mathrm{P}<0.01$ and ${ }^{\# \# \#} \mathrm{P}<0.001$ vs. AC; ${ }^{\boldsymbol{\Delta}} \mathrm{P}<0.05,{ }^{\boldsymbol{\Delta}} \mathrm{P}<0.01$ and ${ }^{\boldsymbol{\Delta} \Delta \boldsymbol{\Delta}} \mathrm{P}<0.001$ vs. YM. 5-FU, 5-fluorouracil; CK-MB, creatine kinase isoenzyme; cTnI, cardiac troponin I; CVF, collagen volume fraction; YC, young age control group; YM, young age model group; AC, aging control group; AM, aging model group; HWI, heart weight index.

group exhibited weight loss from day 9, with the difference in weight loss significantly lower than that of the YM group (Fig. 2A). In addition, HWI evaluation was conducted, but due to 5-FU-induced gastrointestinal toxicity, the weight of the rats in the model group was significantly reduced compared with the control groups, which may have led to inaccurate results; therefore, the heart weight comparisons were combined to facilitate a comprehensive assessment. The results indicated that the heart weight and HWI of AM groups demonstrated an increasing trend compared with the control groups, although the increase in heart weight was not significant in the YM group. 5-FU may have induced structural changes, such as hypertrophy and dilatation of the myocardium, and induced more serious myocardial injury in aging rats (Fig. 2B and C).

In order to observe whether 5-FU-induced cardiotoxicity was time-dependent, blood was collected from the tail vein on days 0,7 and 14 post-5-FU administration, and CK-MB and cTnI detection was conducted (Fig. 2D and E). Serum cTnI values increased on days 7 and 14 following 5-FU treatment in both the YM and AM groups compared with those in the corresponding control groups; this increase was more significant in the AM group (Fig. 2D). Furthermore, the CK-MB level trend was consistent with that of cTnI, although the YM group only demonstrated an increasing trend on day 7 of 5-FU administration, and there was no significant difference compared with the YC group (Fig. 2E). The index of CK-MB growth demonstrated a significant upward trend with prolonged 5-FU administration. However, by day 14 , the CK-MB values of the $\mathrm{AM}$ and $\mathrm{YM}$ groups were no longer significantly different. In conclusion, following prolonged administration of 5-FU, both the serum CK-MB levels and cTnI of rats in the 5-FU model group demonstrated an increasing trend compared with those in the corresponding control groups, and the corresponding detection indices of the AM group were all greater compared with those of the YM group.

The H\&E staining results revealed enlargement of the myofibrillar interstitium and the muscle fiber gap of the YM and AM groups compared with the corresponding control groups, and inflammatory cell infiltration was observed in the myocardial interstitium (Fig. 2G). These pathomorphological effects were the most prominent in the AM group. Masson's staining further demonstrated that the myocardial tissues of the YM and AM groups were irregularly arranged, and that 

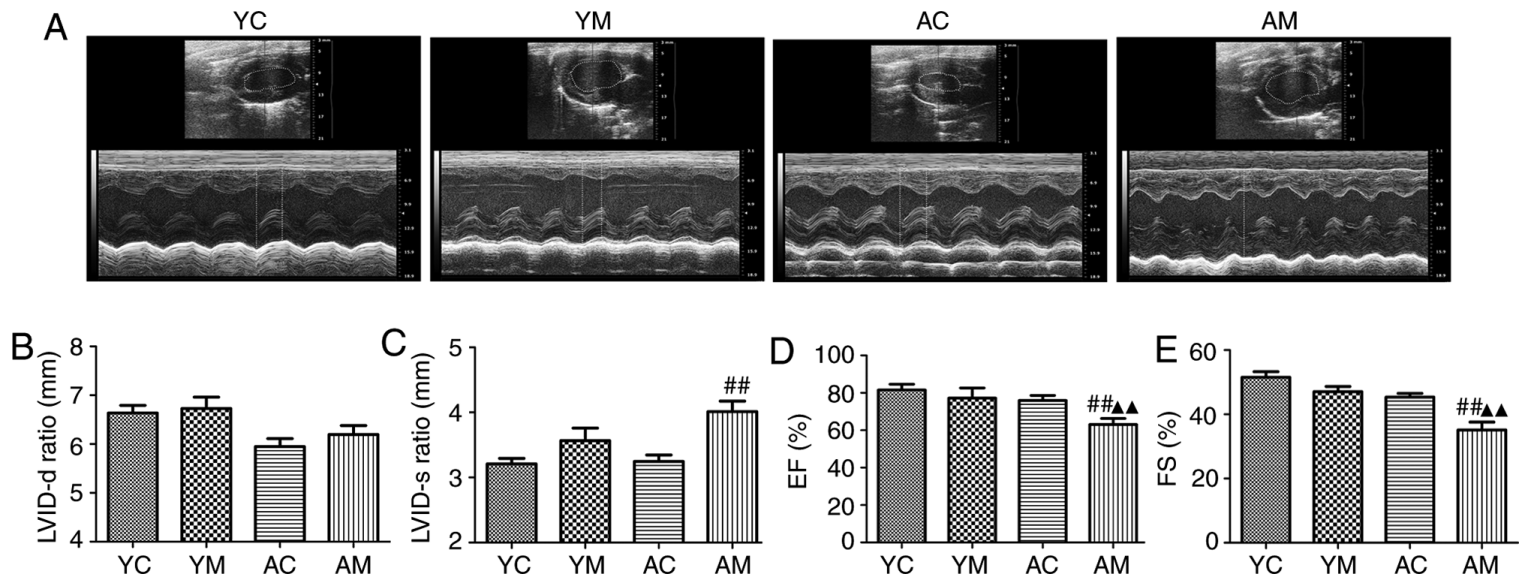

Figure 3. 5-Fluorouracil induces ventricular enlargement and myocardial systolic dysfunction in rats. (A) Representative m-mode echocardiograms for each group of rats. Bar charts demonstrating (B) the LVID-d ratio, (C) the LVID-s ratio, (D) LVEF and (E) LVFS ( $\mathrm{n}=6$ ). ${ }^{\# \#} \mathrm{P}<0.01 \mathrm{vs}$. AC; ${ }^{\mathbf{4}} \mathrm{P}<0.01 \mathrm{vs}$. YM. LVID-d, left ventricular internal dimension in diastole; LVID-s, left ventricular internal dimension in systole; LVEF, left ventricular ejection fraction; LVFS, left ventricular shortening fraction; YC, young age control group; YM, young age model group; AC, aging control group; AM, aging model group.
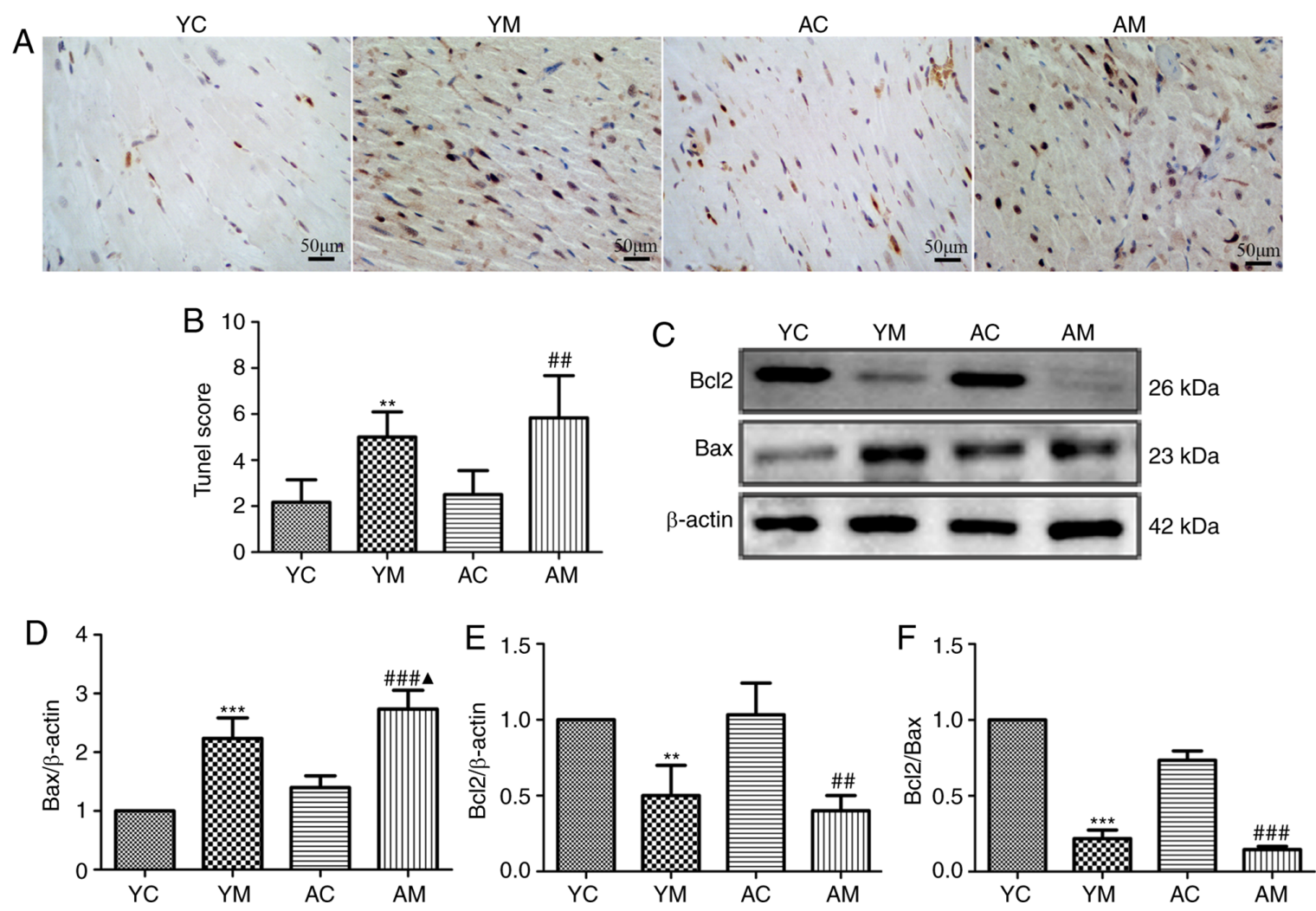

Figure 4. 5-Fluorouracil induces cardiomyocyte apoptosis in rats. (A) Apoptosis in rat myocardial tissues stained with DAPI-TUNEL (n=6; magnification, $\mathrm{x} 200$ ). Brown staining represents apoptotic cells. (B) Apoptosis index (TUNEL score) of rat myocardial tissues $(\mathrm{n}=6)$. (C) Western blot analysis was performed to determine the expression changes of Bcl-2 and Bax proteins ( $\mathrm{n}=3$ ). Evaluation of (D) Bax, (E) Bcl-2 normalized to $\beta$-actin and (F) Bcl-2/Bax expression levels. ${ }^{* *} \mathrm{P}<0.01$ and ${ }^{* * * *} \mathrm{P}<0.001$ vs. YC; ${ }^{\# \#} \mathrm{P}<0.01$ and ${ }^{\# \# \#} \mathrm{P}<0.001$ vs. AC; ${ }^{\wedge} \mathrm{P}<0.05$ vs. $\mathrm{YM}$. YC, young age control group; YM, young age model group; AC, aging control group; AM, aging model group.

the myocardial interstitial tissue was slightly fibrotic in the YM group. By contrast, in the AM group, the myocardial fibers were thickened with increased spacing between fibers, and the blue-stained interstitial area was increased compared with YM group, which was consistent with the H\&E staining results (Fig. 2G). The CV results indicated that the CVF of the $\mathrm{YM}$ and $\mathrm{AM}$ groups were significantly increased, compared with the corresponding control groups (Fig. 2F).
5-FU induces ventricular enlargement, decreases myocardial contractile function and decreases LVEF in aged rats. Previous studies have demonstrated that 5-FU-induced cardiac injury mainly occurs due to a decrease in myocardial systolic function (23-25). Left ventricular structure and function is a suitable indicator to assess the systolic and diastolic function of the heart. In addition, the low volume allows for adequate fixation, dehydration, transparency and staining of the tissue, 
A
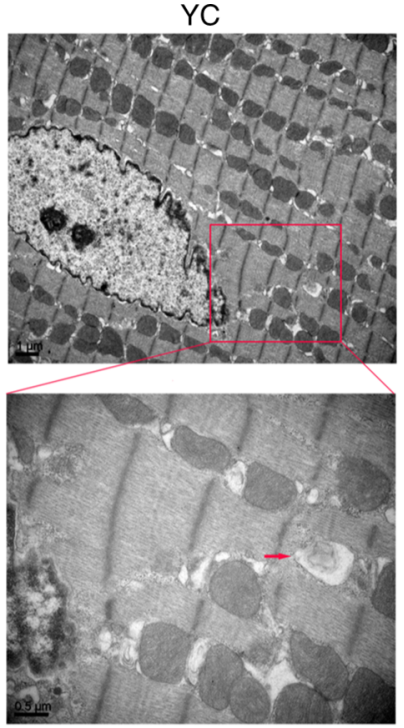

B

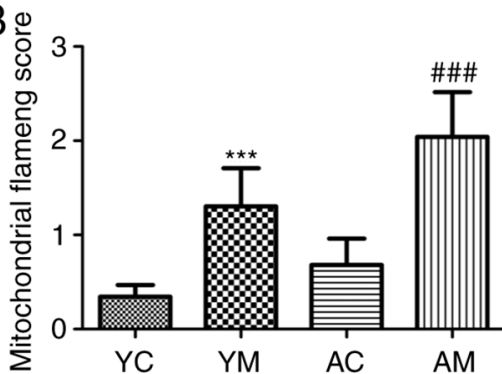

YM
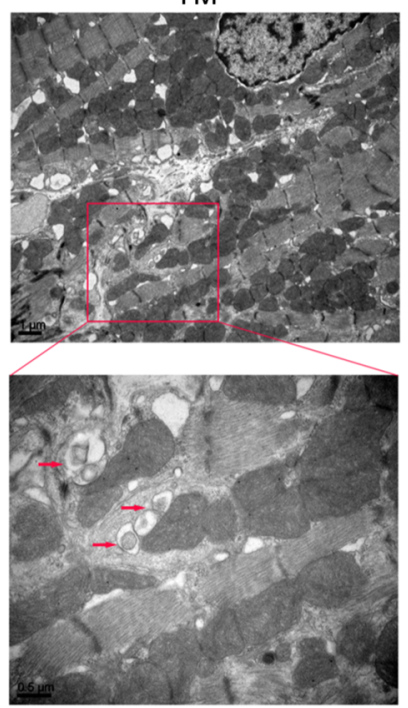

$\mathrm{AC}$
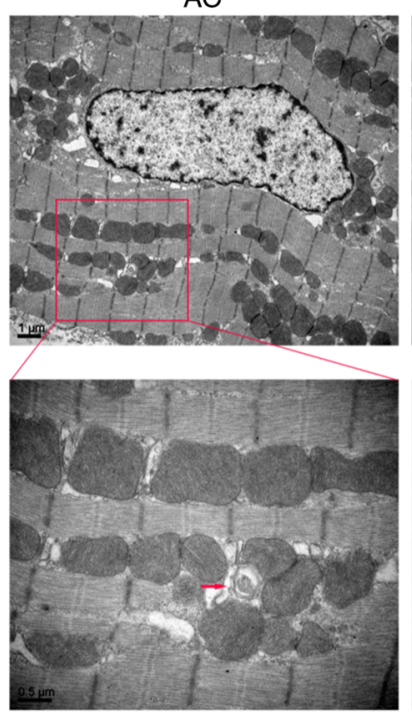

AM
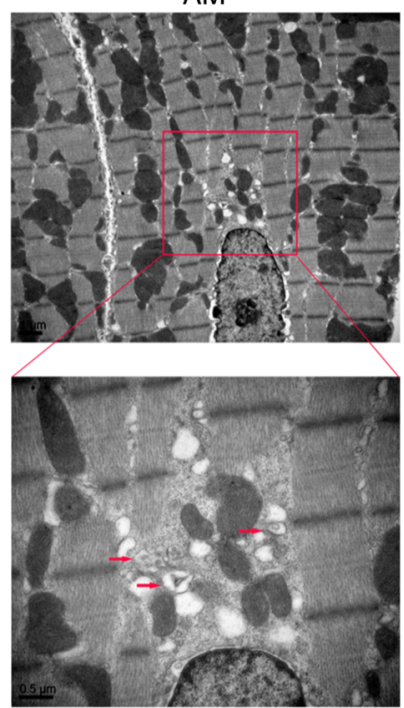
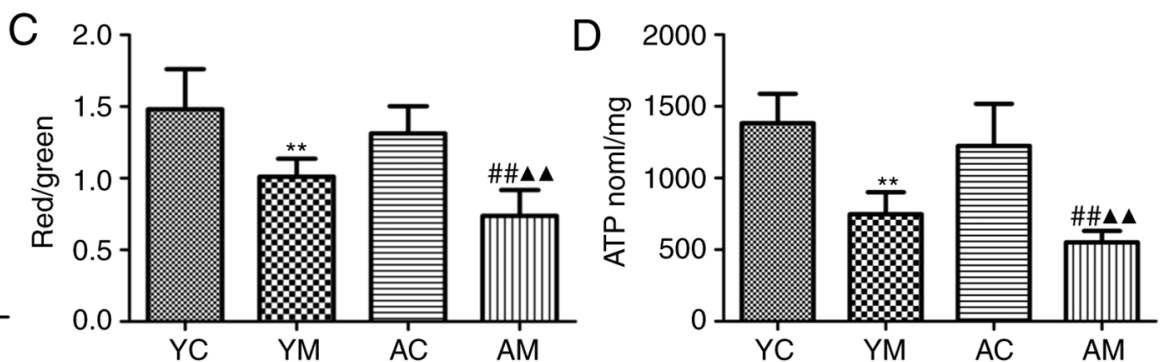

Figure 5. 5-Fluorouracil induces myocardial mitochondrial damage in rats. (A) The ultrastructure of the myocardium was examined by electron microscopy (magnification, $\mathrm{x} 10,000$ and $\mathrm{x} 20,000 ; \mathrm{n}=3$ ). (B) Flameng score for mitochondrial damage. (C and D) mitochondrial membrane potential and ATP contents were assessed in myocardial tissues $(\mathrm{n}=6) .{ }^{* *} \mathrm{P}<0.01$ and ${ }^{* * *} \mathrm{P}<0.001$ vs. $\mathrm{YC} ;{ }^{\# \#} \mathrm{P}<0.01$ and ${ }^{\# \# \#} \mathrm{P}<0.001$ vs. AC; ${ }^{\wedge \boldsymbol{\Delta}} \mathrm{P}<0.01$ vs. YM. YC, young age control group; $\mathrm{YM}$, young age model group; AC, aging control group; AM, aging model group.

avoiding the creation of slits in the sections and ensuring that pathological changes can be assessed. Therefore, in the present study, only the left ventricle of rats was selected for histopathological analysis.

Echocardiography was used to evaluate the changes in cardiac structure and function. Compared with those in the control group, LVID-d and LVID-s in the rat hearts demonstrated an increasing trend following 5-FU treatment, whereas LVEF and LVFS demonstrated a decreasing trend. However, in the YM group, there were no significant differences in LVID-d, LVID-s and LVEF compared with those in the YC group (Fig. 3). These results confirmed that 5-FU induced ventricular enlargement, decreased myocardial contractile function and decreased the LVEF in aged rats. Compared with $\mathrm{AC}$ and YM groups, the induced cardiac structure and LVEF were more severely impaired in aged rats.

5-FU induces cardiomyocyte apoptosis in rats. Apoptosis and autophagy share a number of common signaling pathways and regulatory proteins, such as the bcl-2 family of proteins, caspases, ATG proteins and P53 (26,27). Bcl-2 proteins serve a key dual regulatory role between apoptosis and autophagy (28). In the present study, the levels of apoptosis and the expression levels of apoptosis-related proteins were determined in order to analyze the effects of 5-FU on myocardial cell apoptosis. The results of the TUNEL assay demonstrated that cells in the YM, AC and AM groups exhibited varying degrees of cell hyperchromemia and nuclear fragmentation compared with those in the YC group (Fig. 4A), and nuclear staining of the YM and AM group samples was increased. The apoptotic index of the YM and AM groups was significantly higher compared with that of the YC and AC groups, and was higher in the AM compared with the YM group. The apoptotic index of the AC group appeared to be increased compared with that of the YC group, although the difference was not significant (Fig. 4B).

The results of the western blot analysis demonstrated that the expression levels of the antiapoptotic protein $\mathrm{Bcl}-2$ were decreased in the YM and AM groups compared with those in the YC and AC groups. The expression levels of the proapoptotic protein Bax were increased in the YM and AM groups compared with those in the $\mathrm{YC}$ and $\mathrm{AC}$ groups, respectively, and were significantly increased in the AM group compared with those in the YM group. Furthermore, compared with those in the AC and YM groups, the Bcl-2/Bax ratio was the most notably reduced in the AM group (Fig. 4C-F). The results of the present study suggested that the 5-FU-induced cardiomyocyte apoptosis may be accompanied by autophagy.

5-FU induces myocardial mitochondrial damage in rats. In order to investigate whether mitochondrial functional injury was involved in the mechanism underlying the 5-FU-induced myocardial toxicity, changes in MMP and ATP levels were determined in rat myocardial tissues. The results demonstrated 

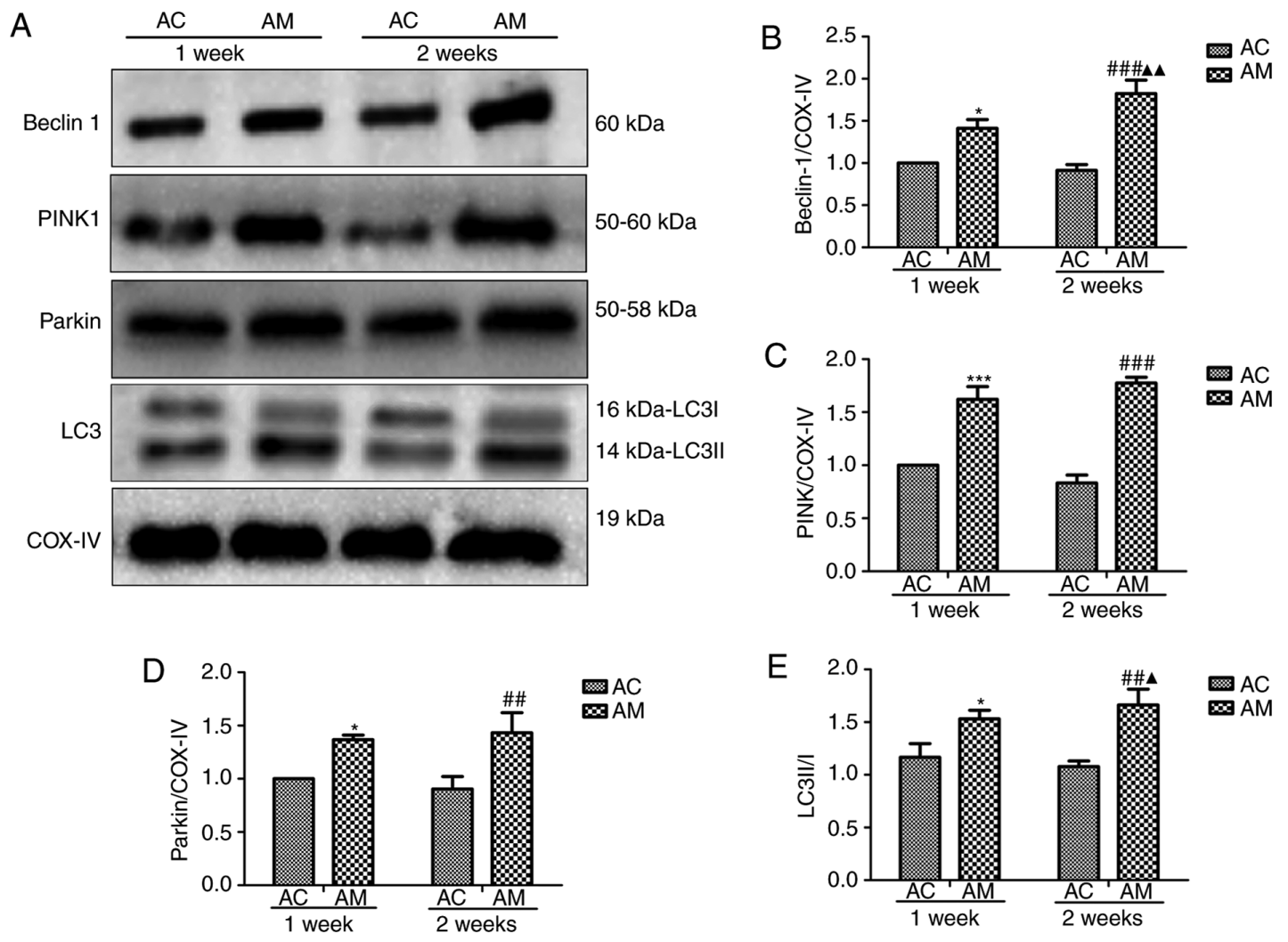

Figure 6. 5-Fluorouracil enhances mitochondrial autophagy in rats. (A) Western blot analysis was performed to determine the expression changes of beclin-1, PINK-1 and Parkin expression levels, and the expression levels of LC3/II relative to those of LC3/I (n=3). (B-E) Evaluation of beclin-1, PINK-1, Parkin and LC3II/I expression levels ( $\mathrm{n}=3)$. ${ }^{*} \mathrm{P}<0.05$ and ${ }^{* * * *} \mathrm{P}<0.001$ vs. the AC-1 week; ${ }^{\# \#} \mathrm{P}<0.01$ and ${ }^{\# \# \#} \mathrm{P}<0.001$ vs. AC; ${ }^{\mathbf{\Delta}} \mathrm{P}<0.05$ and ${ }^{\boldsymbol{\Delta}}{ }^{\boldsymbol{\Delta}} \mathrm{P}<0.01$ vs. the AM-2 weeks. AC, aging control group; AM, aging model group; PINK-1, PTEN-induced putative kinase protein 1; LC3, microtubule-associated protein 1A/1B-light chain 3; COX-IV, cytochrome c oxidase subunit 4.

that 5-FU stimulation significantly reduced mitochondrial MMP and ATP levels in YM and AM groups rats compared with the corresponding control groups, and the ATP level was significantly decreased in the AM group compared with YM group (Fig. 5C and D). These results further confirmed that 5-FU induced mitochondrial energy metabolism disorder in the myocardium, with more severe damage in the AM group compared with the YM group.

Electron microscopy was subsequently used to observe the mitochondrial structure in the rat myocardial tissue. In the YC group, the mitochondrial structure was normal, with a single autophagosome observed. In the AC group, the mitochondria were sparse, however, the structure and arrangement were normal. However, in the YM group, mitochondrial swelling, partial fusion, ridge breakage and an increased number of autophagosomes were observed. In the AM group, the mitochondria were sparse with a condensed structure, ridge fracture, increased lipid droplet deposition and a greater number of autophagosomes compared with the $\mathrm{AC}$ and $\mathrm{YM}$ groups (Fig. 5A).

The semi-quantitative scoring of mitochondrial damage revealed that following 5-FU administration, compared with the corresponding control groups, myocardial mitochondrial damage was more severe in the YM and AM groups, and compared with the YM group, the degree of damage was the most severe in the AM group (Fig. 5B). The results of the present study suggested that 5-FU induced myocardial mitochondrial injury and may enhance mitochondrial autophagy.
5-FU enhances mitochondrial autophagy in rats. In the present study, mitochondrial damage was revealed to induce mitochondrial autophagy and 5-FU caused more serious myocardial damage in aging rats. Thus, 5-FU was predicted to induce excessive mitochondrial autophagy in aging rats, and the degree of autophagy may be associated with the duration of 5-FU exposure. Therefore, the expression of autophagy-related proteins on days 7 and 14 of 5-FU administration was quantitatively analyzed by western blotting (Fig. 6A). COX-IV is a common internal reference gene located in the mitochondria and is commonly used as a mitochondrial loading control. Compared with the 1 week-AC group, 5-FU treatment upregulated the levels of PINK-1, Parkin and beclin-1 expression in the 2 weeks-AM groups, and increased the LC3II/I ratio. In addition, the protein expression levels of beclin-1 and LC3II/I in the 2 weeks group were significantly higher compared with those in the 1-week group (Fig. 6B-E). These results indicated that 5-FU induced myocardial mitochondrial autophagy, and that autophagy was increased with prolonged 5-FU administration. Therefore, the pathological mechanisms underlying 5-FU cardiotoxicity may involve the induction of myocardial damage through the promotion of mitochondrial autophagy.

\section{Discussion}

Considerable cardiotoxicity may occur during 5-FU chemotherapy, with high levels of toxicity observed in the elderly and patients with prior cardiovascular disease $(7,8)$. Therefore, 
detailed investigation is required into the prevention and treatment of cardiac toxicity in elderly patients, focusing on the damaging effects of chemotherapeutic drugs.

The pathogenesis of 5-FU-induced cardiotoxicity is multifactorial, although mitochondrial injury is a potential initiating mechanism $(29,30)$. According to the pharmacological mechanism of 5-FU, F-citrate, a metabolite of 5-FU, acts on the mitochondria to block the tricarboxylic acid cycle, reduce the production of ATP and alter mitochondrial membrane permeability, resulting in mitochondrial dysfunction (14). Mitochondrial damage leads to the progressive accumulation of defective organelles, which further induces cell and tissue damage, resulting in the initiation of mitochondrial autophagy (31). Autophagy is a programmed intracellular degradation process that initiates autophagosome formation by encapsulating degraded macromolecules (32). These autophagosomes fuse with lysosomes for digestion to meet cell metabolic needs, promote organelle renewal and cell homeostasis (32). Although it remains controversial whether the induction of autophagy may be detrimental to myocardial tissue, the activation of autophagy is generally considered to be cardioprotective. However, excessive autophagy results in cell death and myocardial damage $(33,34)$. Therefore, in the present study, the 5-FU-induced dynamic changes to myocardial and mitochondrial injury were investigated in aging rats, and the varying trends of mitochondrial autophagy in 5-FU-induced myocardial injury were discussed.

Cardiotoxic drugs act directly on cardiomyocytes, and myocardial cTnI is a protein only expressed by atrial and ventricular myocytes $(35,36)$. When cardiomyocytes are damaged, cardiac troponin (cTn) is released into the blood, and its levels are proportional to the area and degree of myocardial cell injury (37). In the present study, dynamic changes in myocardial enzyme levels, namely CK-MB and cTnI, were detected in young and aged rats prior to $5-\mathrm{FU}$ treatment as well as on days 7 and 14 of 5-FU administration. The results of the present study demonstrated increasing trends in CK-MB and cTnI levels on day 7 of 5-FU administration in the YM and AM groups, and the levels of these two indicators continued to increase with prolonged 5-FU administration. However, cTnI exhibited a rapid rise within 7 days of 5-FU administration, compared with the rising trend of CK-MB occurring later in the 5-FU administration period. These results suggested that $5-\mathrm{FU}$ induced myocardial injury in rats, and the degree of injury increased with the accumulation of the drug. Furthermore, the degree of damage was more severe in aging rats.

Previous studies have reported that 5-FU-induced cardiac damage results in decreased myocardial contractility and, in severe cases, congestive heart failure (23). In the present study, 5-FU was also demonstrated to induce the expansion of the rat ventricle, and decrease myocardial contractility and LVEF. However, no significant changes in the cardiac structure and function were observed. This may indicate that the 5-FU administration time was too short, or that the dosage was too low to cause cardiac dysfunction. However, 5-FU also induces intestinal mucosal damage $(38,39)$. Preliminary experiments demonstrated that following prolonged duration of 5-FU administration or increasing the administered dosage, there were significant gastrointestinal events, which resulted in increased mortality. Therefore, previously used doses and methods (intraperitoneal (i.p.) injection of $25 \mathrm{mg} / \mathrm{kg} 5-\mathrm{FU}$ every other day for 1 or 2 consecutive weeks of 5-FU administration were used to investigate cardiac injury (30).

In order to verify the 5-FU-induced myocardial mitochondrial damage, changes in myocardial mitochondrial ATP levels and MMP were assessed in rats after 14 days of 5-FU administration in the present study. The results demonstrated that in the YM and AM groups, the myocardial MMP was significantly decreased compared with that in the respective control groups, with more notable effects in the AM group. MMP is a sensitive indicator of the integrity of mitochondrial function, and the dissipation of MMP prevents cells from synthesizing sufficient ATP to complete normal physiological activities $(40,41)$. In the present study, the ATP levels in each group followed the same trend as the levels of MMP. Eskandari et al (12) have reported that a high concentration of 5-FU results in damage beyond the compensation function of the mitochondria, resulting in a reduction in ATP production. These results were consistent with those of the current study. In addition, the ultrastructure of the myocardial mitochondria was observed using electron microscopy. The mitochondria in the YM group exhibited myofibril rupture, swelling and crest fracture or fusion, as well as a high number of autophagosomes. The AM group exhibited mitochondrial sparseness, shrinking mitochondrial structure and a breaking crest, as well as high lipid droplet deposition and autophagosome formation. The results of the present study revealed abnormal morphological changes in the mitochondria and confirmed that 5-FU induced abnormal mitochondrial ultrastructure in rats, resulting in a decline or loss of mitochondrial function.

Mitochondrial damage is associated with cardiomyocyte apoptosis, necrosis and autophagy (28). Autophagy, also termed type II programmed cell death, is involved in the pathogenesis of many diseases, such as heart-related disease (42), cancer (43), vascular dementia (44) and chronic respiratory disease (45). The complex interaction between autophagy and apoptosis cooperatively regulates cell death, and studies have indicated that both may be induced by the same external stimuli $(46,47)$. A study by Pattingre et al (48) has revealed that in the treatment of breast and colon tumors, apoptosis and autophagy are concurrently upregulated, and the use of 3-methyladenine to inhibit autophagy also inhibits caspase activation and reduces apoptosis. In these cases, interaction was apparent between apoptosis and autophagy, and both processes exhibited complementary cooperation (48).

A number of common signaling pathways and regulatory proteins exist between apoptosis and autophagy $(28,49)$. These include the Bcl-2 family of proteins, which play a key dual regulatory role between both processes (48). Beclin-1 directly regulates autophagy and apoptosis by binding to $\mathrm{Bcl}-2$ anti-apoptotic proteins. The Bcl-2/Bcl-XL complex inhibits the activation of autophagy by beclin-1. However, when Bcl-2 competitively binds Bcl-2/Bcl-XL, beclin-1 is released, which induces autophagy (49). Furthermore, Lindqvist et al (50) have determined that the antiapoptotic proteins Bcl-2 and Bcl-XL indirectly inhibit Bax and Bcl-2 homologous antagonist/killer. In summary, apoptosis and autophagy are complex, closely associated processes, and $\mathrm{Bcl}-2$ proteins serve an important 
role in dynamically regulating and maintaining the balance between them (48). In the present study, the expression levels of Bax and Bcl-2 were detected in the myocardial tissues of model rats following 5-FU administration, and the results demonstrated that the expression levels of Bax were decreased in the YM and AM groups, while the expression levels of Bcl-2 were increased compared with those in the corresponding control groups. Thus, we hypothesized that 5-FU-induced myocardial cell apoptosis may be accompanied by autophagy.

Mitochondrial autophagic homeostasis is considered to be an effective and indispensable factor for the mitochondrial maintenance of cellular homeostasis (51). To a certain extent, the maintenance of mitochondrial health depends on mitochondrial dynamics, which involves sufficient, but not excessive autophagy $(18,52)$. When this balance is disrupted, it induces excessive autophagy and aggravate damage. Through animal and clinical experimentation, Eisenberg et al (53) demonstrated that spermidine may serve a protective role in the heart by inducing stable autophagy. However, other studies have reported that excessive mitochondrial autophagy results in impaired autophagosome clearance, which directly promotes cardiac cell death $(54,55)$.

Mitochondrial quality control is necessary to maintain normal cellular activity, for which mitochondrial autophagy is crucial (56). PINK-1/Parkin is a regulatory signaling pathway involved in mitochondrial autophagy $(57,58)$. Previous studies have confirmed that in a healthy state, PINK-1 degrades through the actions of presenilins-associated rhomboid-like protein $(59,60)$. However, during mitochondrial damage, hypoxia and external stimulation, PINK-1 is stabilized and recruits Parkin ligase to initiate autophagy $(59,60)$. Mitochondrial membrane proteins induce autophagy junction protein aggregation through the ubiquitination of Parkin $(59,60)$. Autophagic junction protein binds to LC3 through the LC3-interacting region, and forms LC3II through further lipidation, participating in the completion stage of phagocytic vesicle membrane formation $(61,62)$. Therefore, the conversion from LC3I to LC3II suggests an increase in mitochondrial autophagy flux (62). The increased expression of LC3II may indicate an increase in mitochondrial autophagy; however, autophagosome clearance may be impaired (63). In order to determine the effects of 5-FU-induced mitochondrial autophagy, mitochondrial autophagy-related protein expression changes were examined in the present study 7 and 14 days after 5-FU administration. The expression levels of mitochondrial autophagy-related proteins PINK-1, Parkin, beclin-1 and LC3 were increased on days 7 and 14 of 5-FU administration. In addition, the expression levels of beclin-1 and LC3II were significantly higher in the 2-weeks group compared with those in the 1-week group. Thus, the results of the present study demonstrated that 5-FU induced excessive mitochondrial autophagy and decreased the scavenging ability of damaged mitochondria, which induced myocardial injury. These pathological observations were more prominent in aging rats.

The results of previous studies have suggested that activation of autophagy rescues myocardial injury $(64,65)$. However, autophagy needs to be maintained within a specific level to be beneficial, as aforementioned. The results of the present analysis were inconsistent with the results of previous studies. We hypothesized that the effects of moderate and excessive autophagy may be different within the organism; for example, an appropriate level of autophagy may facilitate the survival of cells, whereas excessive autophagy may promote cell death. Additionally, autophagy is a dynamic process, and moderate autophagy of the myocardial mitochondria may serve a protective role in the early stage of 5-FU intervention. In the present study, with prolonged administration of 5-FU, mitochondrial autophagy was increased, and excessive autophagy induced or exacerbated myocardial injury. Further studies should focus on observing the extent of mitochondrial autophagy on a daily basis, either by electron microscopy or by detection of autophagy-related proteins. However, frequent sampling and testing is not possible in animal experiments; thus, in vitro experiments are required to complement the animal experiments.

Mitochondrial division inhibitor 1 (Mdivi-1) inhibits mitochondrial autophagy, which reduces mitochondrial and myocardial damage (65). Further in vitro experiments, Mdivi-1 and autophagy activator ras-related protein will be used to reveal the dynamic changes in autophagic processes in 5-FU-induced myocardial injury and the potential signaling pathways involved. Future studies will also further investigate whether inhibiting mitochondrial autophagy reduces 5-FU-induced mitochondrial damage, which may promote the development of novel therapeutic strategies for the prevention and reduction of 5-FU-induced myocardial injury.

In conclusion, the results of the present study demonstrated that autophagy was acutely activated following 5-FU-induced myocardial injury. Consistent 5-FU administration induced excessive autophagy in damaged mitochondria, and an impaired ability of autophagosome clearance resulted in direct myocardial cell damage, exacerbating cardiac dysfunction over time.

\section{Acknowledgements}

Not applicable.

\section{Funding}

This research was supported by the Graduate Students Innovation Fund of Tianjin University of Traditional Chinese Medicine (grant no. ZXYCXLX201801), Tianjin Binhai New Area Health Committee Science and Technology Key Project (grant no. 2019BWKZ004), Tianjin Union Medical Center Scientific Research Project (grant no. 2019YJZD001) and the Special Basic Research Project Cooperation in Beijing, Tianjin and Hebei (grant no. 19JCZDJC63900).

\section{Availability of data and materials}

The datasets used and/or analyzed during the current study are available from the corresponding author on reasonable request.

\section{Authors' contributions}

LW supervised and designed the study, and revised and approved the manuscript. YL conceived and designed the study, performed the experiments and data analysis, and wrote the manuscript. XLi and YZ performed the experiments and 
translated the manuscript. XZ co-designed the study and acquired funding. XLe analyzed the animal ultrasound results. LW and YL confirm the authenticity of all the raw data. All authors have read the final manuscript.

\section{Ethics approval and consent to participate}

The animal study was reviewed and approved by The Animal Care and Use Committee of Tianjin Union Medical Center, Tianjin, China (2020-B03).

\section{Patient consent for publication}

Not applicable.

\section{Competing interests}

The authors declare that they have no competing interests.

\section{References}

1. McQuade RM, Stojanovska V, Bornstein JC and Nurgali K: Colorectal cancer chemotherapy: The evolution of treatment and new approaches. Curr Med Chem 24: 1537-1557, 2017.

2. Li C, Ngorsuraches S, Chou C, Chen L and Qian J: Risk factors of fluoropyrimidine induced cardiotoxicity among cancer patients: A systematic review and meta-analysis. Crit Rev Oncol Hematol 162: 103346, 2021.

3. Kastl S, Brunner T, Herrmann O, Riepl M, Fietkau R, GrabenbauerG,Sauer R,Hohenberger W and KleinP:Neoadjuvant radio-chemotherapy in advanced primarilynon-resectable carcinomas of the pancreas. Eur J Surg Oncol 26: 578-582, 2000.

4. Abbruzzese JL and Levin B: Treatment of advanced colorectal cancer. Hematol Oncol Clin North Am 3: 135-153, 1989.

5. Chang HM, Moudgil R, Scarabelli T, Okwuosa TM and Yeh ETH: Cardiovascular complications of cancer therapy: Best practices in diagnosis, prevention, and management: Part 1. J Am Coll Cardiol 70: 2536-2551, 2017.

6. Raber I, Warack S, Kanduri J, Pribish A, Godishala A, Abovich A, Orbite A, Dommaraju S, Frazer M, Peters ML and Asnani A: Fluoropyrimidine-associated cardiotoxicity: A retrospective case-control study. Oncologist 25: e606-e609, 2020.

7. Polk A, Vaage-Nilsen M, Vistisen $K$ and Nielsen DL: Cardiotoxicity in cancer patients treated with 5-fluorouracil or capecitabine: A systematic review of incidence, manifestations and predisposing factors. Cancer Treat Rev 39: 974-984, 2013.

8. Kanduri J, More LA, Godishala A and Asnani A Fluoropyrimidine-associated cardiotoxicity. Cardiol Clin 37 339-405, 2019

9. Abdel-Rahman O: 5-Fluorouracil-related cardiotoxicity; findings from five randomized studies of 5-fluorouracil-based regimens in metastatic colorectal cancer. Clin Colorectal Cancer 18: 58-63, 2019.

10. Sorrentino MF, Kim J, Foderaro AE and Truesdell AG: 5-Fluorouracil induced cardiotoxicity: Review of the literature. Cardiol J 19: 453-458, 2012.

11. Lamberti M, Porto S, Marra M, Zappavigna S, Grimaldi A, Feola D, Pesce D, Naviglio S, Spina A, Sannolo N and Caraglia M: 5-Fluorouracil induces apoptosis in rat cardiocytes through intracellular oxidative stress. J Exp Clin Cancer Res 31: 60, 2012.

12. Eskandari MR, Moghaddam F, Shahraki J and Pourahmad J: A comparison of cardiomyocyte cytotoxic mechanisms for 5 -fluorouracil and its pro-drug capecitabine. Xenobiotica 45 : 79-87, 2015.

13. Focaccetti C, Bruno A, Magnani E, Bartolini D, Principi E, Dallaglio K, Bucci EO, Finzi G, Sessa F, Noonan DM and Albini A: Effects of 5-fluorouracil on morphology, cell cycle, proliferation, apoptosis, autophagy and ROS production in endothelial cells and cardiomyocytes. PLoS One 10: e0115686, 2015.

14. Lemaire L, Malet-Martino MC, Forni M, Martino R and Lasserre B: Cardiotoxicity of commercial 5-fluorouracil vials stems from the alkaline hydrolysis of this drug. Br Cancer 66: $119-127,1992$
15. Polk A, Vistisen K, Vaage-Nilsen M and Nielsen DL: A systematic review of the pathophysiology of 5-fluorouracil-induced cardiotoxicity. BMC Pharmacol Toxicol 15: 47, 2014

16. Mizushima N: A brief history of autophagy from cell biology to physiology and disease. Nat Cell Biol 20: 521-527, 2018.

17. Ong SB, Kalkhoran SB, Cabrera-FUentes HA and Hausenloy DJ: Mitochondrial fusion and fission proteins as novel therapeutic targets for treating cardiovascular disease. Eur J Pharmacol 763: 104-114, 2015.

18. Dombi E, Mortiboys H and Poulton J: Modulating mitophagy in mitochondrial disease. Curr Med Chem 25: 5597-5612, 2018.

19. Yu YL, Wang MS, Wang ZS, Yu JQ, Song YY, Li BZ, Hong ZZ, Wen ZN, Xu GS, Liang SJ, et al: A guidebook for the care and use of laboratory animals. In: Chinese society for the laboratory animal science Publ. Taiwan, pp64-75, 2004.

20. Zou YH, Xu ZW, Huang R, Chen LM, Yu L, Wang X, Wang SC, Guo XJ, Zhang W, Cai ZZ, et al: Laboratory animal science. In: Science Press Publ. Beijing, pp200-203, 2016.

21. Soslow RA, Dannenberg AJ, Rush D, Woerner BM, Khan KN, Masferrer J and Koki AT: COX-2 is expressed in human pulmonary, colonic, and mammary tumors. Cancer 89: 2637-2645, 2000.

22. Flameng W, Borgers M, Daenen W and Stalpaert G: Ultrastructural and cytochemical correlates of myocardial protection by cardiac hypothermia in man. J Thora Cardiovasc Surg 79: 413-424, 1980.

23. Mishra T, Shokr M, Ahmed A and Afonso L: Acute reversible left ventricular systolic dysfunction associated with 5-fluorouracil therapy: A rare and increasingly recognised cardiotoxicity of a commonly used drug. BMJ Case Rep 12: e230499, 2019.

24. Iskandar MZ, Quasem W and El-Omar M: 5-Fluorouracil cardiotoxicity: Reversible left ventricular systolic dysfunction with early detection. BMJ Case Rep 2015: bcr2015209347, 2015.

25. Depetris I, Marino D, Bonzano A, Cagnazzo C, Filippi R, Aglietta $M$ and Leone F: Fluoropyrimidine-induced cardiotoxicity. Crit Rev Oncol Hematol 124: 1-10, 2018.

26. Gump JM and Thorburn A: Autophagy and apoptosis: What is the connection? Trends Cell Biol 21: 387-392, 2011.

27. Su M, Mei Y and Sinha S: Role of the crosstalk between autophagy and apoptosis in cancer. J Oncol 2013: 102735, 2013.

28. Chen Q, Kang J and Fu C: The independence of and associations among apoptosis, autophagy, and necrosis. Signal Transduct Target Ther 3: 18, 2018.

29. Sara JD, Kaur J, Khodadadi R, Rehman M, Lobo R, Chakrabarti S, Herrmann J, Lerman A and Grothey A: 5-Fluorouracil and cardiotoxicity: A review. Ther Adv Med Oncol 10: 1758835918780140, 2018.

30. Zhang D and Ma J: Mitochondrial dynamics in rat heart induced by 5-fluorouracil. Med Sci Monit 24: 6666-6672, 2018.

31. Roca-Agujetas V, de Dios C, Lestón L, Marí M, Morales A and Colell A: Recent insights into the mitochondrial role in autophagy and its regulation by oxidative stress. Oxid Med Cell Longev 2019: 3809308, 2019.

32. Yu L, Chen Y and Tooze SA: Autophagy pathway: Cellular and molecular mechanisms. Autophagy 14: 207-215, 2018.

33. Maejima Y: The critical role of autophagy in heart failure. Nihon Yakurigaku Zasshi 151: 100-105, 2018 (In Japanese).

34. Sridhar S, Botbol Y, Macian F and Cuervo AM: Autophagy and disease: Always two sides to a problem. J Pathol 226: 255-273, 2012.

35. Novo G, Cadeddu C, Sucato V, Pagliaro P, Romano S, Tocchetti CG, Zito C, Longobardo L, Nodari S and Penco M: Role of biomarkers in monitoring antiblastic cardiotoxicity. J Cardiovasc Med (Hagerstown) 17 (Suppl 1): S27-S34, 2016.

36. Jones M, O'Gorman P, Kelly C, Mahon N and Fitzgibbon MC: High-sensitive cardiac troponin-I facilitates timely detection of subclinical anthracycline-mediated cardiac injury. Ann Clin Biochem 54: 149-157, 2017.

37. Mokhtar AT, Begum J, Buth KJ and Legare JF: Cardiac troponin $\mathrm{T}$ is an important predictor of mortality after cardiac surgery. J Crit Care 38: 41-46, 2017.

38. Rtibi K, Selmi S, Grami D, Amri M, Sebai H and Marzouki L: Contribution of oxidative stress in acute intestinal mucositis induced by 5 fluorouracil (5-FU) and its pro-drug capecitabine in rats. Toxicol Mech Methods 28: 262-267, 2018

39. McQuade RM, Stojanovska V, Donald E, Abalo R, Bornstein JC and Nurgali K: Gastrointestinal dysfunction and enteric neurotoxicity following treatment with anticancer chemotherapeutic agent 5-fluorouracil. Neurogastroenterol Motil 28: 1861-1875, 2016 . 
40. Murphy E, Ardehali H, Balaban RS, DiLisa F, Dorn GW II, Kitsis RN, Otsu K, Ping P, Rizzuto R, Sack MN, et al: Mitochondrial function, biology, and role in disease: A scientific statement from the American heart association. Circ Res 118: 1960-1991, 2016.

41. Basu Ball W, Neff JK and Gohil VM: The role of nonbilayer phospholipids in mitochondrial structure and function. FEBS Lett 592: 1273-1290, 2018.

42. Sciarretta S, Maejima Y, Zablocki D and Sadoshima J: The role of autophagy in the heart. Annu Rev Physiol 80: 1-26, 2018.

43. Onorati AV, Dyczynski M, Ojha R and Amaravadi RK: Targeting autophagy in cancer. Cancer 124: 3307-3318, 2018.

44. Wang W, Qiao O, Ji H, Zhang X, Han X, Zhang Y, Wang J, Li X and Gao W: Autophagy in vascular dementia and natural products with autophagy regulating activity. Pharmacol Res 170: 105756, 2021.

45. Racanelli AC, Kikkers SA, Choi AMK and Cloonan SM: Autophagy and inflammation in chronic respiratory disease. Autophagy 14: 221-232, 2018.

46. Mukhopadhyay S, Panda PK, Sinha N, Das DN and Bhutia SK: Autophagy and apoptosis: Where do they meet? Apoptosis 19: 555-566, 2014.

47. Young MM, Kester M and Wang HG: Sphingolipids: Regulators of crosstalk between apoptosis and autophagy. J Lipid Res 54: 5-19, 2013.

48. Pattingre S, Tassa A, Qu X, Garuti R, Liang XH, Mizushima N, Packer M, Schneider MD and Levine B: Bcl-2 antiapoptotic proteins inhibit beclin 1-dependent autophagy. Cell 122: 927-939, 2005.

49. Nakajima S, Aikawa C, Nozawa T, Minowa-Nozawa A, Toh $\mathrm{H}$ and Nakagawa I: Bcl-xL affects group a streptococcus-induced autophagy directly, by inhibiting fusion between autophagosomes and lysosomes, and indirectly, by inhibiting bacterial internalization via interaction with beclin 1-UVRAG. PLoS One 13: e0170138, 2017

50. Lindqvist LM, Heinlein M, Huang DC and Vaux DL: Prosurvival Bcl-2 family members affect autophagy only indirectly, by inhibiting Bax and Bak. Proc Nati Acad Sci USA 111: 8512-8517, 2014

51. Haeussler S, Köhler F, Witting M, Premm MF, Rolland SG, Fischer C, Chauve L, Casanueva O and Conradt B: Autophagy compensates for defects in mitochondrial dynamics. PLOS Genet 16: e1008638, 2020.

52. Yoo SM and Jung YK: A molecular approach to mitophagy and mitochondrial dynamics. Mol Cells 41: 18-26, 2018.

53. Eisenberg T, Abdellatif M, Schroeder S, Primessnig U, Stekovic S, Pendl T, Harger A, Schipke J, Zimmermann A, Schmidt A, et al: Cardioprotection and lifespan extension by the natural polyamine spermidine. Nat Med 22: 1428-1438, 2016.
54. Lin XL, Xiao WJ, Xiao LL and Liu MH: Molecular mechanisms of autophagy in cardiac ischemia/reperfusion injury (review). Mol Med Rep 18: 675-683, 2018.

55. Liu CY, Zhang YH, Li RB, Zhou LY, An T, Zhang RC, Zhai M, Huang Y, Yan YW, Dong YH, et al: LncRNA CAIF inhibits autophagy and attenuates myocardial infarction by blocking p53-mediated myocardin transcription. Nat Commun 9: 29, 2018.

56. Ng MYW, Wai T and Simonsen A: Quality control of the mitochondrion. Dev Cell 56: 881-905, 2021.

57. Hamacher-Brady A and Brady NR: Mitophagy programs: Mechanisms and physiological implications of mitochondrial targeting by autophagy. Cell Mol Life Sci 73: 775-795, 2016.

58. Tanaka K: The PINK1-Parkin axis: An overview. Neurosci Res 159: 9-15, 2020.

59. Chun Y and Kim J: Autophagy: An essential degradation program for cellular homeostasis and life. Cells 7: 278, 2018.

60. Kawajiri S, Saiki S, Sato S, Sato F, Hatano T, Eguchi H and Hattori N: PINK1 is recruited to mitochondria with parkin and associates with LC3 in mitophagy. FEBS Lett 584: 1073-1079, 2010.

61. Sciarretta S, Yee D, Nagarajan N, Bianchi F, Saito T, Valenti V, Tong M, Del Re DP, Vecchione C, Schirone L, et al: Trehalose-induced activation of autophagy improves cardiac remodeling after myocardial infarction. J Am Coll Cardiol 71: 1999-2010, 2018

62. Kabeya Y, Mizushima N, Yamamoto A, Oshitani-Okamoto S, Ohsumi Y and Yoshimori T: LC3, GABARAP and GATE16 localize to autophagosomal membrane depending on form-II formation. J Cell Sci 117: 2805-2812, 2004.

63. Wu X, Qin Y, Zhu X, Liu D, Chen F, Xu S, Zheng D, Zhou Y and Luo J: Increased expression of DRAM1 confers myocardial protection against ischemia via restoring autophagy flux. J Mol Cell Cardiol 124: 70-82, 2018.

64. Liang H, Su X, Wu Q, Shan H, Lv L, Yu T, Zhao X, Sun J, Yang R, Zhang L, et al: LncRNA 2810403D21Rik/Mirf promotes ischemic myocardial injury by regulating autophagy through targeting Mir26a. Autophagy 16: 1077-1691, 2020.

65. Dong Y, Undyala VVR and Przyklenk K: Inhibition of mitochondrial fission as a molecular target for cardioprotection: Critical importance of the timing of treatment. Basic Res Cardiol 111: $59,2016$.

This work is licensed under a Creative Commons Attribution-NonCommercial-NoDerivatives 4.0 International (CC BY-NC-ND 4.0) License. 